\title{
The multi-faceted synergy between Swift and Fermi in radio-loud AGN studies
}

\author{
F. D'Ammando*i \\ INAF - Istituto di Radioastronomia, Via Gobetti 101, I-40129 Bologna, Italy \\ Dip. di Fisica e Astronomia, Universitá di Bologna, Viale B. Pichat 6/2, I-40127 Bologna, Italy \\ E-mail: dammando@ira.inaf.it
}

\begin{abstract}
Since its launch in 2008 June, the Fermi Gamma-ray Space Telescope has opened a new era in high-energy astrophysics. The unprecedented sensitivity, angular resolution and effective area of the Large Area Telescope on board Fermi, together with the nearly continuous observation of the entire $\gamma$-ray sky assures a formidable opportunity to study in detail $\gamma$-ray emitting AGN of various types. In this context the Swift satellite, thanks to its broad band coverage and scheduling flexibility, creates a perfect synergy with Fermi.

Swift and Fermi coordinated monitoring campaigns of radio-loud AGN allowed us to investigate correlated variability at different frequencies and to build time-resolved spectral energy distributions from optical to $\gamma$-rays, constraining the emission mechanisms at work in these objects. The rapid Swift follow-up observations of $\gamma$-ray flaring AGN detected by Fermi-LAT were also fundamental in firmly associating the $\gamma$-ray sources with their low-energy counterparts. We present some interesting results obtained from Fermi-LAT and Swift observations of $\gamma$-ray flaring AGN in the first six years of Fermi operation.
\end{abstract}

Swift: 10 Years of Discovery,

2-5 December 2014

La Sapienza University, Rome, Italy

\footnotetext{
${ }^{*}$ Speaker.

†n behalf of the Fermi Large Area Telescope Collaboration
} 


\section{Introduction}

Only a small percentage of Active Galactic Nuclei (AGN) are radio-loud, and this characteristic is commonly ascribed to the presence of a relativistic jet, roughly perpendicular to the accretion disc. These jets are engines able to carry out a huge amount of power, not only in the form of radiation but especially in the form of kinetic energy and magnetic fields. Understanding the structure and dynamics of relativistic jets is an essential step for the comprehension and interpretation of many phenomenological properties of AGN. In addition to blazars and radio galaxies [1], recently relativistic jets were discovered also in narrow-line Seyfert 1 galaxies (e.g. [2, 3]).

Multifrequency observations are a powerful tool for studying relativistic jets in radio-loud AGN. The Fermi satellite with its continuous observation of the entire $\gamma$-ray sky and high sensitivity assures a formidable opportunity to have a complete census of the extragalactic $\gamma$-ray sky, opening a window to correlated investigations of radio-loud AGN over the entire electromagnetic spectrum. Thanks to its broad band coverage and scheduling flexibility, the Swift satellite creates a perfect synergy with Fermi covering the electromagnetic spectrum from optical to $\gamma$-rays. In this paper we present some examples of the multi-faced synergy between Swift and Fermi in radio-loud AGN.

\section{Long-term monitoring of AGN}

Long-term observations with Swift and Fermi, in conjunction with other facilities in different bands are crucial for monitoring different flux levels of variable AGN and investigate if the spectral and flux variability properties are significantly different in high and low state. The study of correlated multiwavelength variability allows us to achieve a better understanding of the structure of the inner jet, the origin of the seed photons for the inverse Compton process and the emission mechanisms at work in radio-loud AGN.

The importance of long-term monitoring was confirmed e.g. by the observations of the blazar PKS 0537-441 obtained from microwaves through $\gamma$-rays by SMA, REM, ATOM, Swift and Fermi during 2008 August-2010 April [4]. Strong variability has been observed in $\gamma$-rays, with two major flaring episodes: 2009 July and 2010 March (Fig. 1, left panel). The spectral energy distribution (SED) of the source cannot be modelled by a simple synchrotron self-Compton model, as opposed to many BL Lacs, but the addition of an external Compton component of seed photons from a dust torus is needed. The $230 \mathrm{GHz}$ light curve showed an increase simultaneous with the $\gamma$-ray one, indicating co-spatiality of the $\mathrm{mm}$ and $\gamma$-ray emission region likely at large distance from the central engine. The low, average, and high activity SED of the source could be fit changing only the electron distribution parameters. An overall correlation between the $\gamma$-ray band with the $R$-band and $K$-band has been observed with no significant time lag. On the other hand, when inspecting the light curves on short time scales some differences are evident. In particular, flaring activity has been detected in near-infrared and optical bands with no evident $\gamma$-ray counterparts in 2009 September and November (Fig. 1, left panel). Moderate variability has been observed in X-rays with no correlation between flux and photon index. An increase of the detected X-ray flux with no counterpart at the other wavelengths has been observed in 2008 October, suggesting once more a complex correlation between the emission at different energy bands. 

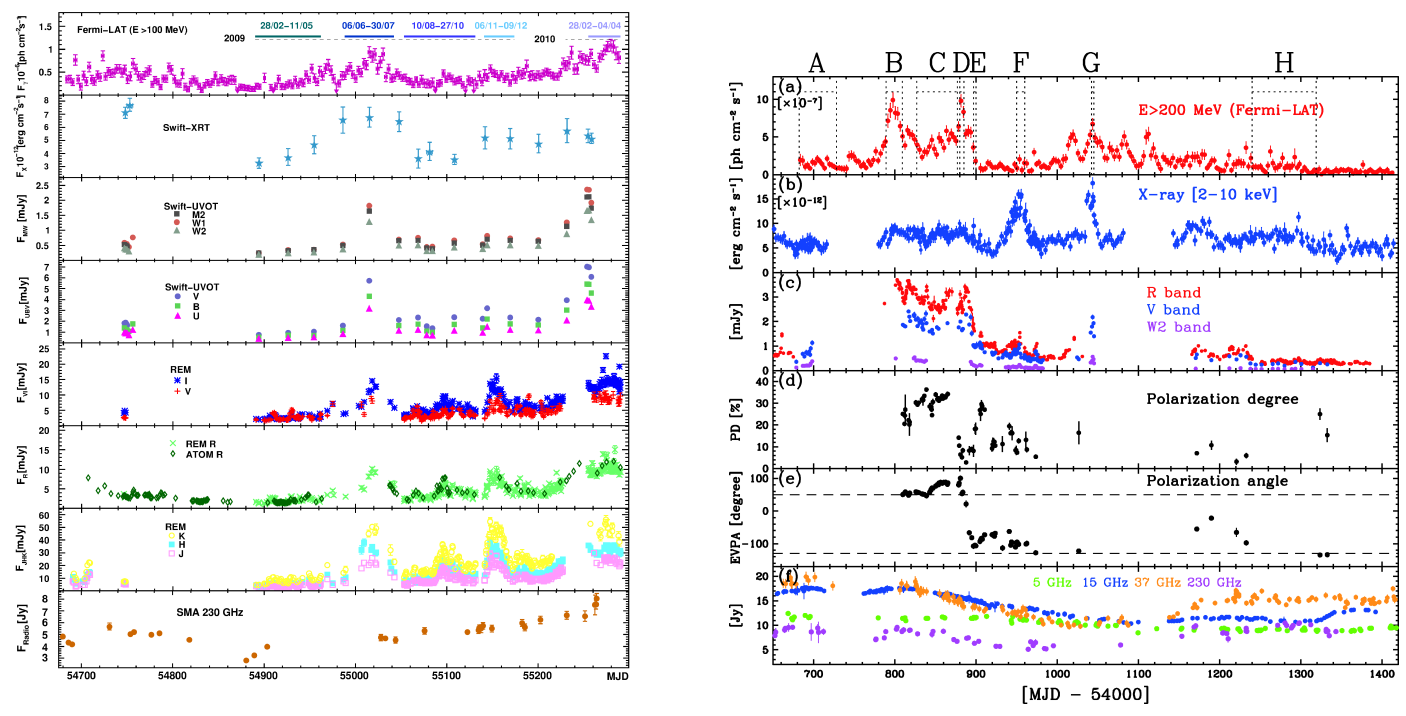

Figure 1: Left Panel: Multifrequency light curve for PKS 0537-441. The period covered is 2008 August 4-2010 April 4. The data sets were collected (from top to bottom) by Fermi-LAT ( $\gamma$-rays), Swift-XRT (0.3$10 \mathrm{keV}$ ), Swift-UVOT (w1, m2, w2, and v, b, u filters), REM (V and I bands), REM and ATOM (R band), REM (J, H, K bands), and SMA (230 GHz). Adapted from [4]. Right Panel: Multi-band light curves of 3C 279 from 2008 August to 2010 August. (a): $\gamma$-ray flux averaged over 3 days. (b): X-ray flux measured by Swift-XRT and RXTE-PCA. (c): UV-optical fluxes in R band (red), V band (blue) and w2 band (magenta). (d): Polarization degree in the optical band. (e): Polarization angle in the optical band. The horizontal dashed lines refer to the angle of $50 \mathrm{deg}$ and $-130 \mathrm{deg}$. (e): Radio fluxes at $230 \mathrm{GHz}$ (magenta), $37 \mathrm{GHz}$ (orange), $15 \mathrm{GHz}$ (blue) and $5 \mathrm{GHz}$ (green). All X-ray, UV and optical data are corrected for the Galactic absorption. Adapted from [5].

\section{Isolated X-ray flares}

Similar to PKS 0537-441, X-ray observations of 3C 279 during 2008-2010 revealed a pair of pronounced flares separated by $\sim 90$ days, both not contemporaneous to increased activity in optical or $\gamma$-ray bands (Fig. 1, right panel). These isolated X-ray flares may be related to changes of the source parameters such as the jet direction, Lorentz factor, and/or location of the dissipation event, or they may require more unusual solutions: bulk-Compton process, inefficient electron acceleration above a given energy, hadronic processes [5].

\section{Identification of $\gamma$-ray sources}

Variability is common in $\gamma$-ray blazars and provides a powerful tool to unambiguously associate them with objects known at other wavelengths and to study the emission mechanisms at work. When combined with simultaneous ground- and space-based multifrequency observations, the Fermi-LAT achieves its full capability for the identification of the $\gamma$-ray sources with counterparts at lower energies and the knowledge of their emission processes, as reported e.g. in 

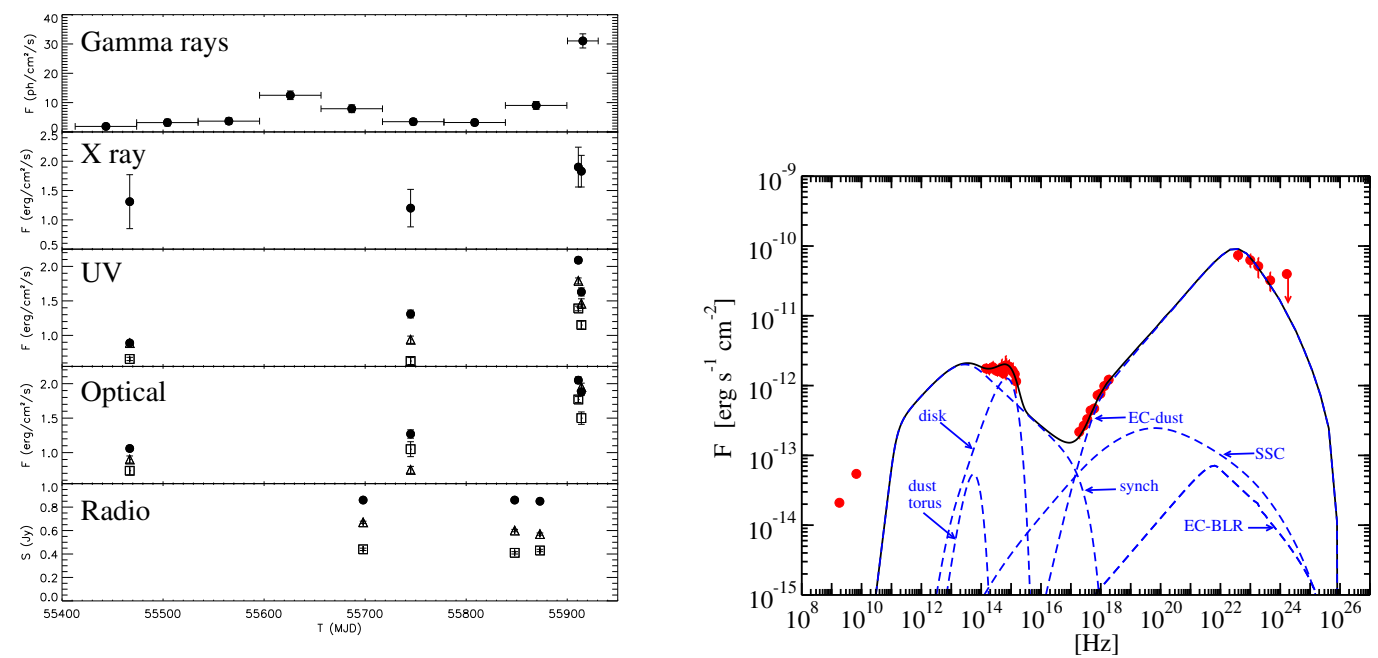

Figure 2: Left panel: light curves of PKS 2123-463 collected in $\gamma$-rays by Fermi-LAT (0.1-100 GeV flux in units of $10^{-8}$ photons $\mathrm{cm}^{-2} \mathrm{~s}^{-1}$ ), in X-rays by Swift/XRT (flux in units of $10^{-12} \mathrm{erg} \mathrm{cm}^{-2} \mathrm{~s}^{-1}$ ), in UV (filters w1: empty squares, m2: empty triangles and w2: filled circles; flux in units of $10^{-12} \mathrm{erg} \mathrm{cm}^{-2} \mathrm{~s}^{-1}$ ) and in optical (filters u: empty squares, b: empty triangles and v: filled circles; flux in units of $10^{-13} \mathrm{erg}$ $\mathrm{cm}^{-2} \mathrm{~s}^{-1}$ ) by Swift/UVOT, and radio by ATCA (empty squares: $40 \mathrm{GHz}$, empty triangles: $17 \mathrm{GHz}$ and filled circle: $5.5 \mathrm{GHz}$ ) between 2010 June and 2011 December. Right panel: SED data (circles and squares) and model fit (solid curve) of PKS 2123-463 with the model components shown as dashed curves. The data points were collected by GROND (2011 December 18), Swift (UVOT and XRT; 2011 December 19) and Fermi-LAT (2011 December 10-19), together with radio data from ATCA (2011 December 19) and KAT-7 (2011 December 24). Adapted from [6].

[6] for PKS 2123-463 and in [7] for TXS 0536+135. In particular, the strict spatial association with the lower energy counterpart together with a simultaneous increase of the activity in optical, UV, X-ray and $\gamma$-ray bands (Fig. 2, left panel) led to a firm identification of the $\gamma$-ray source with PKS 2123-463. We fit the SED with a synchrotron/external Compton model (Fig. 2, right panel). A thermal disc component is necessary to explain the optical/UV emission detected by Swift/UVOT. This disc has a luminosity of about $1.8 \times 10^{46} \mathrm{erg} \mathrm{s}^{-1}$, and a fit to the disc emission assuming a Schwarzschild (i.e., non-rotating) black hole gives a mass of about $2 \times 10^{9} \mathrm{M}_{\odot}$.

\section{Narrow-line Seyfert 1 galaxies}

Thanks to Swift and Fermi-LAT observations, we are able to investigate the broad-band spectra and the correlated variability in different energy bands of this new class of $\gamma$-ray emitting AGN $[8,2,3]$. We compared the SED of SBS $0846+513$ during the flaring state in 2012 May with that of a quiescent state (Fig. 3, left panel). The SED of the two different activity states, modeled by an external Compton component of seed photons from a dust torus, could be fitted by changing the electron distribution parameters as well as the magnetic field [9], consistent with the modeling of 

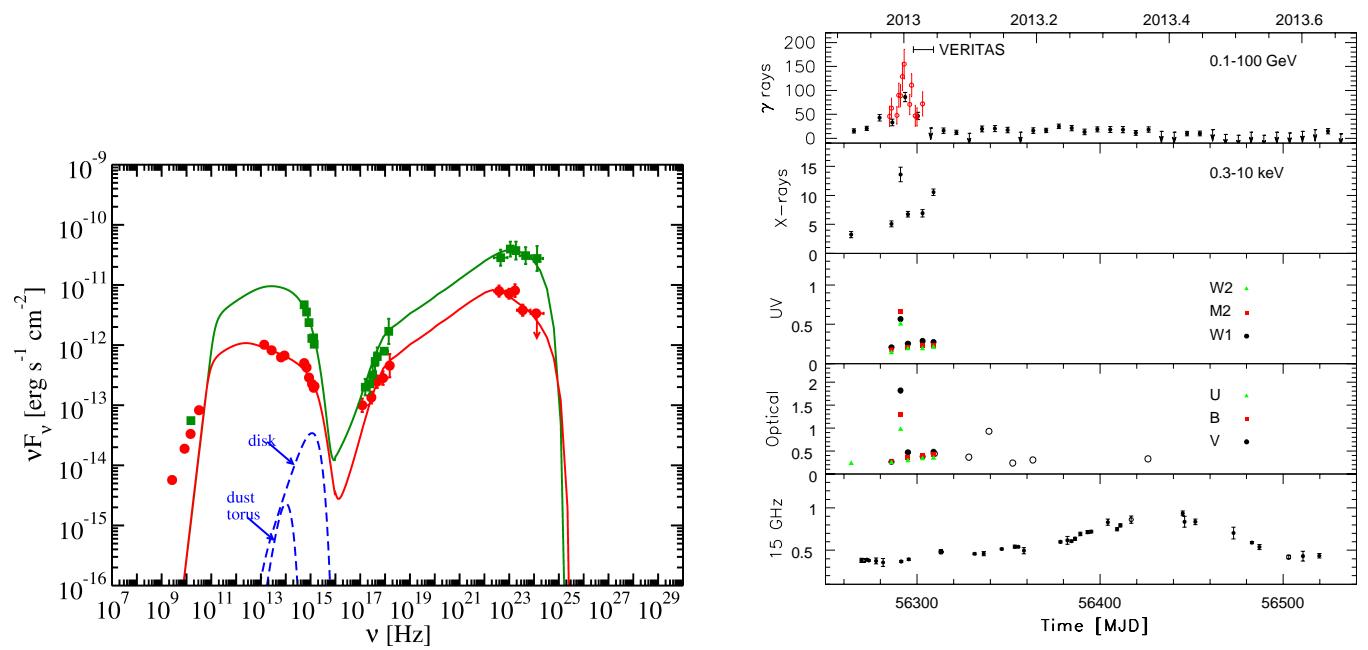

Figure 3: Left panel: SED data (squares) and model fit (solid curve) of SBS 0846+513 in flaring activity with the model components shown as dashed curves. The data points were collected by OVRO 40-m (2012 May 17), Swift (UVOT and XRT; 2012 May 27), and Fermi-LAT (2012 May 20-29). The SED in the quiescent state reported in [3] is shown as circles. Adapted from [9]. Right panel: Multifrequency light curve for PMN J0948+0022. The period covered is 2012 December 1 - 2013 August 31 (MJD 5626256535). The data were collected (from top to bottom) by Fermi-LAT ( $\gamma$-rays; in units of $10^{-8} \mathrm{ph} \mathrm{cm}^{-2} \mathrm{~s}^{-1}$ ), Swift-XRT (0.3-10 keV; in units of $\left.10^{-12} \mathrm{erg} \mathrm{cm}^{-2} \mathrm{~s}^{-1}\right)$, Swift-UVOT ( $w 1, m 2$, and $w 2$ filters; in units of mJy), CRTS (open circles; in units of mJy) and Swift-UVOT ( $v, b$, and $u$ filters; in units of mJy), MOJAVE (open circles) and OVRO (15 GHz; in units of Jy). The horizontal line in the top panel indicates the period of the VERITAS observation. Adapted from [10].

different activity states of PKS 0208-512 [11]. A significant shift of the synchrotron peak to higher frequencies was observed during the 2012 May flaring episode, similar to FSRQ (e.g., PKS 1510089; [12]). Contrary to what is observed in PMN J0948+0022 [13], no significant evidence of thermal emission from the accretion disc has been observed in SBS 0846+513. A complex connection between the radio and $\gamma$-ray emission was observed for SBS 0846+513 and PMN J0948+0022 (Fig. 3, right panel), as discussed in detail e.g. in [9, 14, 13, 15]. At Very High Energy (VHE; E $>100 \mathrm{GeV}$ ), VERITAS observations of PMN J0948+0022 were carried out during 2013 January 6-13, after the $\gamma$-ray flare observed by Fermi-LAT on 2013 January 1 . These observations resulted in an upper limit of $\mathrm{F}_{>0.2 \mathrm{TeV}}<4 \times 10^{-12} \mathrm{ph} \mathrm{cm}^{-2} \mathrm{~s}^{-1}[10]$.

\section{Constraining the BH spin of FSRQ}

The fit of the optical/UV part of the spectrum of the flat spectrum radio quasar $4 \mathrm{C}+21.35$ collected by Swift/UVOT on 2010 April 29 (mainly produced by the accretion disc) seems to favor an inner disc radius of $<6$ gravitational radii, as one would expect from a maximally prograderotating Kerr black hole [16]. 


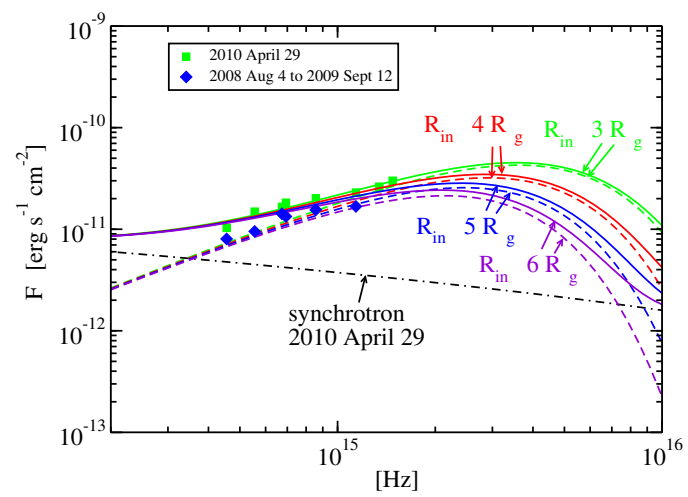

Figure 4: Model disc emission for several inner disc radii are shown (dashed curves), while the synchrotron component from the model fit of 2010 April 29 is shown as the dot-dashed curve. The total (synchrotron + disk) emission is shown as the solid curves. Models for $4 \mathrm{C}+21.35$ with large inner disc radii do not provide an adequate fit to the UV data of 2010 April 29 [16].

\section{Acknowledgments}

The Fermi-LAT Collaboration acknowledges support for LAT development, operation and data analysis from NASA and DOE (United States), CEA/Irfu and IN2P3/CNRS (France), ASI and INFN (Italy), MEXT, KEK, and JAXA (Japan), and the K.A. Wallenberg Foundation, the Swedish Research Council and the National Space Board (Sweden). Science analysis support in the operations phase from INAF (Italy) and CNES (France) is also gratefully acknowledged.

\section{References}

[1] F. Acero, M. Ackermann, M. Ajello, et al., Fermi Large Area Telescope Third Source Catalog, ApJS (2015) in press, [astro-ph. HE/1501.02003]

[2] A. A. Abdo, M. Ackermann, M. Ajello, et al., Radio-loud Narrow-line Seyfert 1 as a new class of gamma-ray active galactic nuclei, ApJ (2009) 707 L142

[3] F. D'Ammando, M. Orienti, J. Finke, et al., SBS 0846+513: a new $\gamma$-ray-emitting narrow-line Seyfert 1 galaxy, MNRAS (2012) 426317

[4] F. D'Ammando, E. Antolini, G. Tosti, et al., Long-term monitoring of PKS 0537-441 with Fermi-LAT and multiwavelength observations, MNRAS (2013) 4312481

[5] M. Hayashida, G. M. Madejski, K. Nalewajko, et al., The Structure and Emission Model of the Relativistic Jet in the Quasar 3C 279 Inferred from Radio to High-energy $\gamma$-Ray Observations in 2008-2010, ApJ (2012) $\mathbf{7 5 4} 114$

[6] F. D'Ammando, A. Rau, P. Schady, et al., PKS 2123-463: a confirmed $\gamma$-ray blazar at high redshift, MNRAS (2012) 427893

[7] M. Orienti, F. D’Ammando, M. Giroletti, et al., Exploring the multiband emission of TXS 0536+145: the most distant $\gamma$-ray flaring blazar, MNRAS (2014) 4443040

[8] A. A. Abdo, M. Ackermann, M., Ajello, et al., Fermi/Large Area Telescope Discovery of Gamma-Ray Emission from a Relativistic Jet in the Narrow-Line Quasar PMN J0948+0022, ApJ (2009) 699976 
[9] F. D'Ammando, M. Orienti, J. Finke, et al., Multifrequency studies of the narrow-line Seyfert 1 galaxy SBS 0846+513, MNRAS (2013) 436191

[10] F. D'Ammando, M. Orienti, J. Finke, et al., The most powerful flaring activity from the NLSyl PMN J0948+O022, MNRAS (2015) 4462456

[11] R. Chatterjee, K. Nalewajko, A. D. Myers, An Optical-Near-infrared Outburst with no Accompanying $\gamma$-Rays in the Blazar PKS 0208-512, ApJ (2013) $736 \mathrm{~L} 11$

[12] F. D'Ammando, C. M. Raiteri, M. Villata, et al., AGILE detection of extreme $\gamma$-ray activity from the blazar PKS 1510-089 during March 2009. Multifrequency analysis, A\&A (2011) 529145

[13] F. D'Ammando, J. Larsson, M. Orienti, et al., Multiwavelength observations of the $\gamma$-ray-emitting narrow-line Seyfert 1 PMN J0948+0022 in 2011, MNRAS (2014) 4383521

[14] M. Orienti, F. D'Ammando, M. Giroletti, et al., On the connection between radio and gamma rays. Variability and polarization properties in relativistic jets, EPJWC (2013) 6104009

[15] L. Foschini, E. Angelakis, L. Fuhrmann, et al., Radio-to- $\gamma$-ray monitoring of the narrow-line Seyfert 1 galaxy PMN J0948+0022 from 2008 to 2011, A\&A (2012) 548 A106

[16] M. Ackermann, M. Ajello, A. Allafort, et al., Multifrequency Studies of the Peculiar Quasar 4C +21.35 during the 2010 Flaring Activity, ApJ (2014) 786157 